\title{
Texture Feature Extraction and Classification of SEM Images of Wheat Straw/Polypropylene Composites in Accelerated Aging Test
}

\author{
Huan Zhang, ${ }^{1,2,3}$ Chunxia He, ${ }^{1}$ Min Yu, ${ }^{1}$ and Jingjing Fu ${ }^{1}$ \\ ${ }^{1}$ College of Engineering, Jiangsu Key Laboratory for Intelligent Agricultural Equipment, Nanjing Agricultural University, \\ Nanjing 210031, China \\ ${ }^{2}$ College of Mechanical and Electronic Engineering, Qingdao Agricultural University, Qingdao 266109, China \\ ${ }^{3}$ Jiangsu Key Laboratory of Large Engineering Equipment Detection and Control, Xuzhou Institute of Technology, \\ Xuzhou 221111, China
}

Correspondence should be addressed to Chunxia He; chunxiahe@tom.com

Received 24 September 2014; Revised 17 April 2015; Accepted 20 April 2015

Academic Editor: Xinfeng Xie

Copyright (C) 2015 Huan Zhang et al. This is an open access article distributed under the Creative Commons Attribution License, which permits unrestricted use, distribution, and reproduction in any medium, provided the original work is properly cited.

\begin{abstract}
Wheat straw/polypropylene composites are green recycled and biomass-based material. After accelerated aging test of the composite was done, practical and effective methods for characterization and extraction of texture feature of microscopic Scanning Electron Microscopy (SEM) images of composites were investigated in this paper, and involved data compression and classification recognition were studied as well. Through Angle Measure Technique (AMT) method, the complexity spectra, MA spectra, of the preprocessed SEM images of the composites were derived and then the first four principal components of MA spectra using Principal Component Analysis (PCA) were extracted accordingly. Two kinds of classifiers based on Extreme Learning Machine (ELM) and Support Vector Machine (SVM) were introduced to classify the SEM images into five different aging periods in this paper. The research results indicate that AMT method is a very novel and effective approach in texture feature characterization and analysis of SEM images of composites and high classification accuracy of SEM images in different aging periods by using intelligent recognition can be reached.
\end{abstract}

\section{Introduction}

Wheat straw/polypropylene composites are a new biomassbased material substituting for the traditional wood/plastics composites (WPCs) in many fields, which can be prepared by using plastic or rubber as matrix and wheat straw plant fiber, a usual agricultural residue, as filler. Compared with the crude wood, these kinds of wood/plastics composites have many advantages, such as better water resistance and anticorrosive property. Although the composites are green, recycled, environmentally friendly material, they would exhibit aging phenomenon unavoidably [1-3]. If the microscopic SEM images of composites are effectively characterized and correctly classified in different aging periods, it is easy to accurately identify the aging stage of the material and to determine the dynamic development of its aging process.
The topics of quantitative characterization and intelligent modeling of microscopic surface topography and internal structure of composite material have been of great importance in recent years; for example, digital image processing of SEM was proposed to study relationship between cracks shape and wearing loss in the preparation of carbon fabricepoxy composite [4]; SEM image processing and analysis was introduced in structural characteristics analysis of anodic porous alumina [5]; SEM microstructure characterization and modeling were researched on the numerical study on internal frost damage of digital cement paste samples with cohesive zone; and so forth [6].

Angle Measure Technique (AMT) method, first proposed by Andrle in 1994, was used for quantitative characterization of curve complexity of geomorphic line [7]. This technique can be considered as a characterization tool of 
TABLE 1: Raw materials of the experiment.

\begin{tabular}{lcr}
\hline Main raw material & Trade name & Origin or manufacturer \\
\hline Polypropylene (film) & - & Jiangyin Oriental Plastic Packaging Co., Ltd., China \\
Wheat straw & - & Tanghuang, Jiangyin, Jiangsu, China \\
Silane coupling agent & KH550 & Shanghai Yaohua Glass Co., Ltd., China \\
Zinc stearate & Industrial grade & Shanghai Yan'an Grease Chemical Plant, China \\
\hline
\end{tabular}

signal complexity through scale domain transform and then was improved and introduced into many fields by some researcher. For instance, Huang and Esbensen used AMT and multivariate data analysis to characterize many types of powders, such as sand, pellet, and rapeseed [8]. Indahl and Næs characterized and built modeling of different sizes of oil droplets in mayonnaise by using AMT and PCR [9]. Kucheryavski investigated discrimination and classification of medical images by PLS-discrimination analysis after using $\mathrm{AMT}$ and wavelet transformation to construct feature vectors [10]. Fongaro and Kvaal studied surface texture characterization and multivariate feature extraction of texture images of Italian pasta by means of Heterogeneity (HTG), gray level cooccurrence matrix (GLCM), AMT, and so forth [11].

In the accelerated aging test, SEM images of the composites in 5 different aging periods were shot, and then AMT spectra of texture feature of microscopic SEM images of composites were derived by AMT method and lowdimension reduced spectra data were extracted by PCA. According to the extracted feature matrix, the microscopic SEM images of composites in different aging periods were recognized and classified based on two kinds of intelligent classifier, Extreme Learning Machine (ELM) and Support Vector Machine (SVM). Our investigation can provide a new way for quantitative characterization and depiction of microscopic surface topography of SEM images of composite material in its aging test and precisely determine the composites' aging stage and indirectly reflect corresponding mechanical, physical, and chemical properties accordingly by using intelligent recognition and classification.

\section{Experimental}

2.1. Materials. Raw materials of sample preparation of wheat straw/polypropylene composites were shown in Table 1.

\subsection{Material Specimen Preparation and Its Accelerated Aging} Test. Wheat straw/polypropylene composites were prepared by mixing molding method first; mass fraction of wheat straw powder, zinc stearate, and polypropylene was $50 \%$, $1.5 \%$, and $48.5 \%$, respectively. Then, the specimen of wheat straw/polypropylene composites was made by hot press molding with the size of $120 \mathrm{~mm} \times 100 \mathrm{~mm} \times 15 \mathrm{~mm}$. According to the Chinese National Standard GB/T16422.31997 UV accelerated aging test for wheat straw/polypropylene composites was conducted. In the aging test, UV-A340 xenon lamp was used, and the temperature of blackboard and condensation was set to $60^{\circ} \mathrm{C}$ and $50^{\circ} \mathrm{C}$, respectively. One aging cycle is $12 \mathrm{~h}$ (including illumination, $8 \mathrm{~h}$, and condensation, $4 \mathrm{~h}$ ), and every 20 cycles were sampled once totaling 100 cycles (i.e., $1200 \mathrm{~h}$ ). The 5 different aging periods, respectively, correspond to the 20th, 40th, 60th, 80th, and 100th aging period [12].

2.3. SEM Images Shoot. Before shooting SEM pictures of composites sample, we need to spray gold on them first. SEM photos of microscopic surface topography of the sample bars were acquired using JSW-6300 Scanning Electron Microscope with accelerating voltage $20 \mathrm{kV}$, and photo magnification is $50 \mathrm{x}, 100 \mathrm{x}$, and $200 \mathrm{x}$, respectively. Then, after carefully observing the typical region of interest, we carefully chose and took four SEM photos of the microscopic surface topography of specimen of composites in five different aging periods with the original size of $1232 \times 912$ pixels for each magnification.

\section{Methodology}

3.1. Image Preprocessing and Its Subsamples Preparation. After microscopic SEM photos of the composites were shot in five different aging periods, ROI (region of interest) images or image subsamples at the same size $(400 \times 400$ pixels $)$ were selected from the SEM raw images at the magnification of $100 \mathrm{x}$ for the following textural feature extraction and analysis based on AMT method. For each aging stage, 16 preprocessed subsamples of the SEM images $(400 \times 400$ pixels) of material specimens were chosen out elaborately for classification prediction (i.e., totally 80 subsamples in five different aging periods).

For the entire samples, cross validation was introduced to help construct predictive models and then to validate the models in this work, through which we can assess how the results of the predictive models will generalize to a separate test dataset subsequently (to keep the classification models from overfitting).

3.2. AMT Method. The calculating procedure of AMT method is described below: for a 1D measurement series or $1 \mathrm{D}$ digital curve (2D measurement series should be converted to $1 \mathrm{D}$ ones first), in each scale factor $s$, one point from a number of random sampling points (usually 500 points for $1 \mathrm{D}$ data series and above $2 \sim 5 \%$ of the total number of pixels for the unfolded imagery) is chosen as the starting point first and also as the center of the circle with the radius $s$ (i.e., the present scale). The circle intersects the $1 \mathrm{D}$ digital curve at 2 points which form an angle with the starting point, taking its supplementary angle into consideration. The calculating angle will change with the location move of the random 


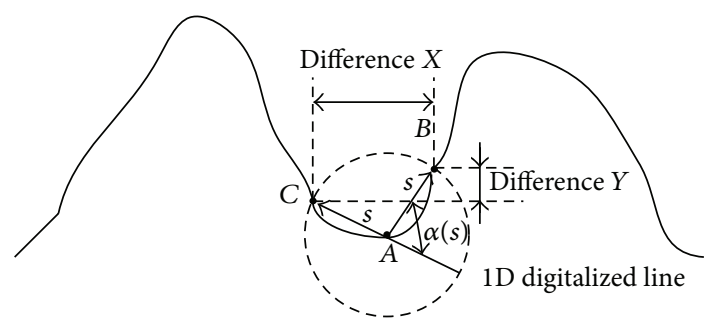

FIGURE 1: Schematic diagram of AMT method.

sampling point in the digital curve at the present scale factor $s$; thereby, a series of angle measuring values will be generated and then averaged into one angle, which is called Mean Angle (MA) or $\alpha(s)$ as the function of the scale factor $s$ [8] (see Figure 1). With continuous increase of the scale factor $s$, the Mean Angle is changing also. The calculating formula of Mean Angle or $\alpha(s)$ according to AMT method is defined as follows:

$$
\mathrm{MA}=\frac{k}{N} \sum_{i=1}^{N}\left(\alpha_{i}\right)_{s}
$$

where $\left(\alpha_{i}\right)_{s}$ is the $i$ th measuring angle at the present scale $s$ and MA or $\alpha(s)$ is the average of $N$ measurement angles; $k$ is a adjusting factor ranged from 0 to 1 which can enlarge the variation in the unfolded $1 \mathrm{D}$ image pixels and it is set to constant 1 in our work.

The production of AMT spectra of SEM images of composites in different aging periods was evaluated using the software ImageJ (NIH, USA, Version 1.46) with AMT plugin script (http://arken.umb.no/ kkvaal/eamtexplorer/ imagej_plugins.html) written by Professor Knut Kvaal, Norwegian University of Life Sciences (NMBU). The main selected parameters of AMT spectra of SEM photos of straw/polypropylene composites were as follows:

(1) Mean Angle measuring is done using AMT linear method.

(2) Minimum scale radius is 1 pixel, maximum scale radius is 500 pixels, and scale increment is 1 pixel.

(3) Total number of random sampling points is 5000 points.

(4) Unfolded type adopts "spiral" (outside to inside way) converting $2 \mathrm{D}$ images to $1 \mathrm{D}$ digitalized curve.

(5) Number of sample pixels to unfold selects all.

(6) Correction option is considered and used to digitize the line, and more information about this choice can be found in [13].

3.3. MA Spectra Extraction Using PCA. AMT spectra (refer to MA spectra commonly used) of SEM images of wheat straw/polypropylene composites in different aging periods have distinct texture features with the extension of material aging cycles (Figure 5), so it is possible to extract the texture characteristics by using AMT method. As the references reported, MA data is a function depending on continuous factor scale $s$, and therefore the exported measuring angles $\alpha(s)$ are high-dimensional data and it is possible that multicollinearity of MA spectra might exist in some cases. Raw AMT spectra seemed to be directly employed into the classifier after simply selecting some scale range, but this job was completed by naked eyes of observer resulting in subjective differences and randomness to some extent and possible time-consuming process sometimes. In addition, scale number in the interval(s) of MA spectra might be relatively large compared to the input of some classifier; for instance, the input number of Artificial Neural Network is a few or a dozen usually. It is important to eliminate possible strong collinearity and at the same time to realize data compression. As a matter of fact, the collinearity existed indeed in the MA spectra by observation intuitively (Figure 6). In consideration of these facts first four principal components (PC1 PC4) were extracted from MA spectra by PCA quickly and automatically in this work. After dimensional reduction the compressed MA spectra data was loaded to input matrix of the two following classifiers for the following analysis.

\subsection{Classification Prediction Using ELM and SVM}

3.4.1. ELM Classification Prediction. ELM is superior to the traditional feed forward neural network in many aspects, such as classification, regression, and artificial intelligence $[14,15]$. As a novel single hidden layer of Artificial Neural Network, it can obtain a unique optimal solution finally by randomly generating connection weights between the input layers and hidden layers and thresholds of hidden layer neurons. The weights of the output layer are calculated according to the following formula:

$$
\widehat{\beta}=H^{+} T^{\prime} \text {, }
$$

where $\mathrm{H}^{+}$is Moore-Penrose generalized inverse matrix.

The input of ELM neutral network is the first four principal components of MA spectra of the SEM image of composites extracted by PCA. Programming of ELM classification prediction of five different aging stages was realized in MATLAB (MathWorks, USA, Version R2010a), and the program of classification includes two main functions, that is, ELM training function and ELM prediction one [16, 17]. Flow chart of ELM algorithm on predicting classification is shown in Figure 2.

3.4.2. SVM Classification Prediction. SVM is a new data mining technology which is very suitable for small sample statistical analysis and can change the nonlinear classification problem into linear classification one by high-dimensional space transformation [18]. Because the aging period classification of wheat straw/polypropylene composites in aging test is a multiclass classification problem, multiclass SVM classification was utilized here. Algorithm programming of SVM classification prediction of SEM images was realized by using Libsvm software package (Libsvm, Version 3.1) developed by Professor Lin Chih-Jen, National Taiwan University, providing interface with SVM MATLAB toolbox. 


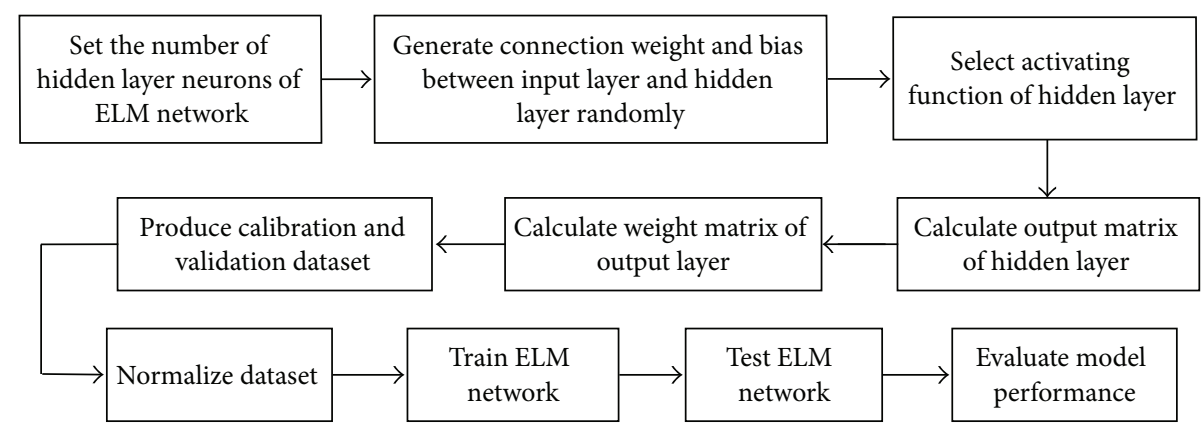

FIGURE 2: Flow chart of ELM algorithm on predicting classification.

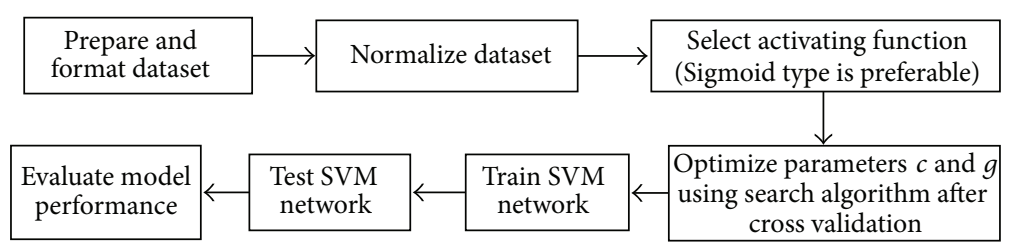

FIGURE 3: Flow chart of SVM algorithm on predicting classification.

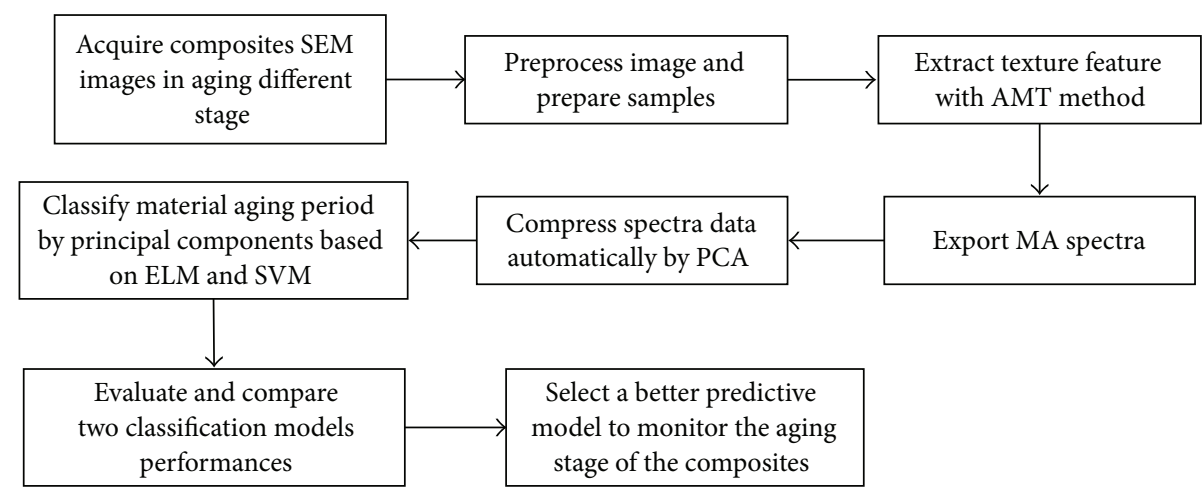

FIGURE 4: System overall diagram of texture feature extraction and classification of SEM images of the composites.

Function SVMcgForClass should be called first when running the program. Using the optimal parameters returned by SVMcgForClass function the classifier model was built and trained through svmtrain function with cross validation procedure; finally svmpredict function with the optimal parameters estimated by the svmtrain function would predict classification results $[18,19]$. Flow chart of SVM algorithm on predicting classification is shown in Figure 3.

System overall diagram of texture feature extraction and classification of SEM images of the composites was given in Figure 4.

\section{Results and Discussion}

\subsection{Results}

4.1.1. Export and Analysis of AMT Spectra of the Composites $S E M$ Images. For each of the five different aging periods, one image sample which has relatively typical characteristics of aging was picked out and illustrated. SEM image samples of the composites in five different aging periods are shown in Figure 5.

From Figure 5, it can be easily found that, with the growth of aging time of straw/polypropylene composites, the aging cracks on the surface of the composites gradually appeared, showing that their length, width, and depth were more and more long, more and more large, and more and more deep. At the end of the aging period, the material's surface was severely eroded, resulting in shedding in a huge area. It is obvious that rather distinct texture features are presented by the development and change of those aging cracks in SEM pictures; in other words, the complexity of SEM images of different aging periods is higher and higher. Mean MA spectra of typical SEM image samples of the composites in five different aging periods are shown in Figure 6.

From Figure 6, it is clearly the case where MA spectra of the five SEM images of the composites lay orderly from bottom to top in most of the range of sale domain according to the extension of aging period; that is, spectrum for 20th aging period is at the bottom, the one for 100th aging period is at 


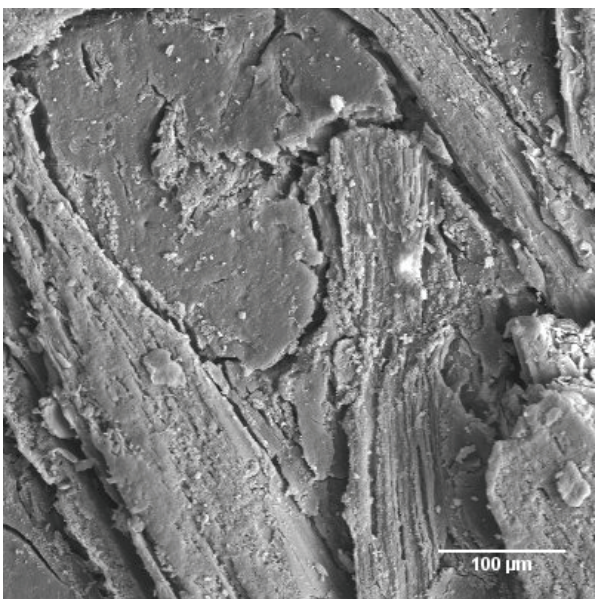

(a)

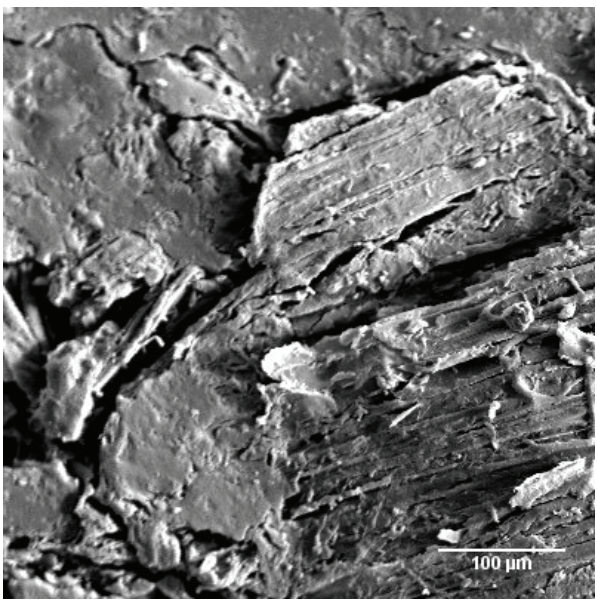

(c)

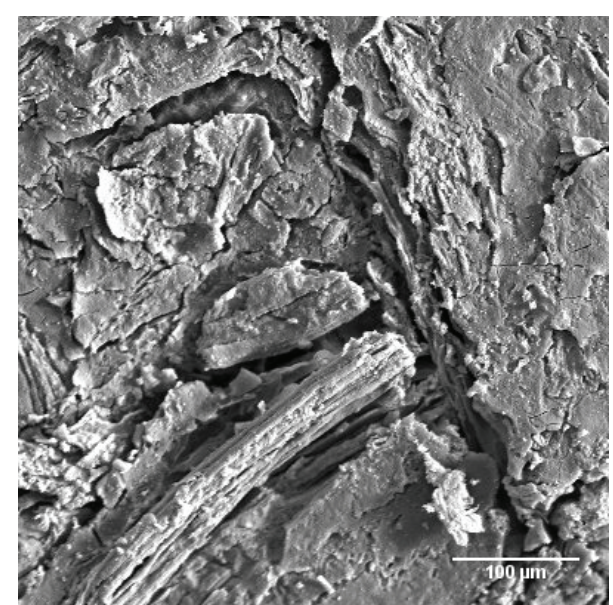

(b)

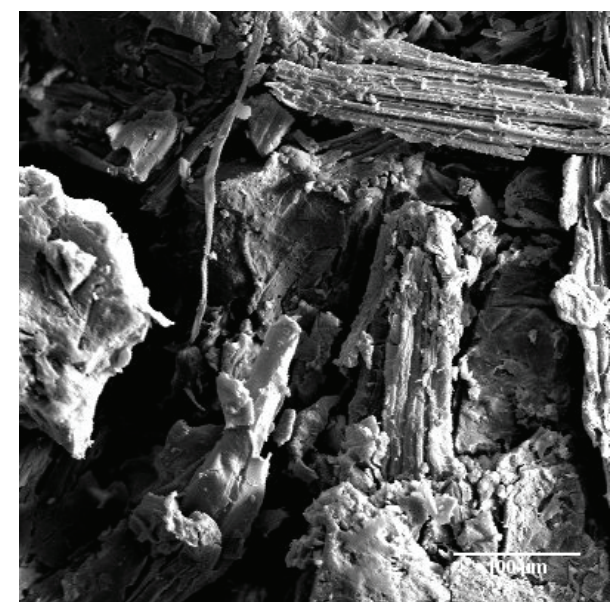

(d)

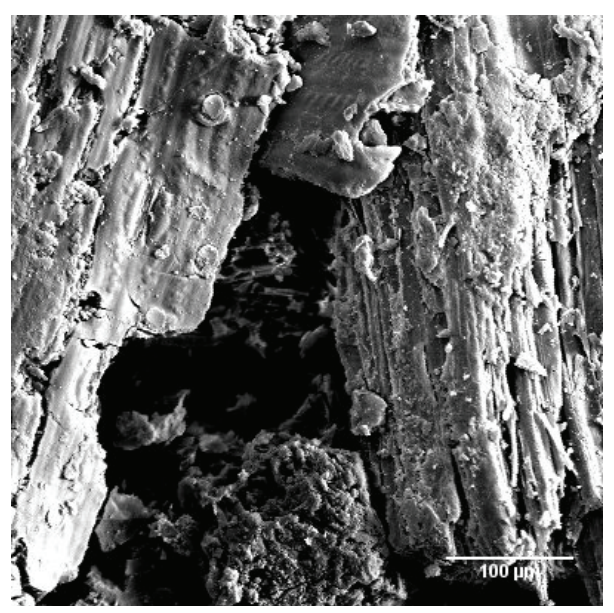

(e)

FIGURE 5: SEM image samples of the composites in 5 different aging periods: (a) in 20th aging period; (b) in 40th aging period; (c) in 60th aging period; (d) in 80th aging period; and (e) in 100th aging period. 

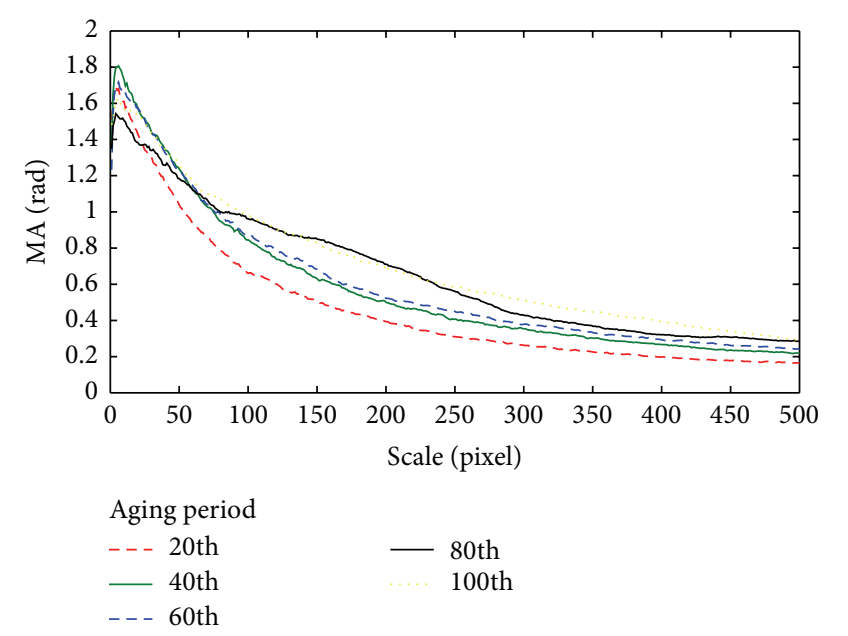

FIGURE 6: MA spectra of SEM image samples of the composites in 5 different aging periods.
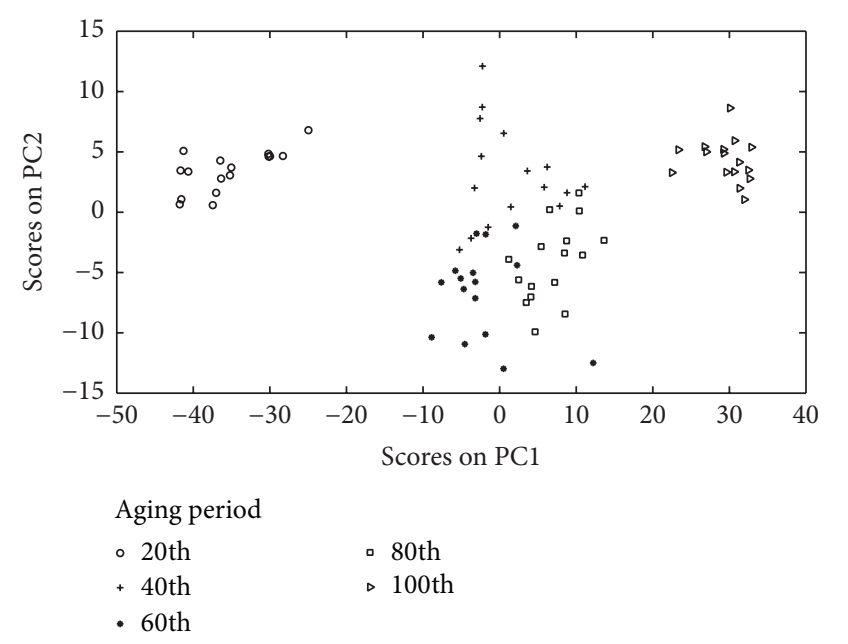

Figure 7: PCA scores plot of AMT spectra of SEM image samples of the composites in five different aging periods.

the top, and those for 40th, 60th, and 100th aging period are in the middle, which means that spectrum of 20th aging period has the lowest complexity, while spectrum of 100th aging period has the highest complexity. The spectra correctly characterize and represent the texture complexity of SEM images of the composites in different aging periods. Therefore, it is indicated that AMT spectra have very excellent ability of characterization and discrimination of texture feature of microscopic SEM images of composites in different aging stage. Besides, it can be observed that the collinearity existing among the spectra data ranged approximately from scale 130 to 200 and scale 275 to 500 as those curves in Figure 6 were almost parallel to each other in these two intervals.

PCA analysis was applied to the exported MA spectra of all SEM image subsamples of the composite, and the first two principal components are good enough to explain $97.4 \%$ variance of the samples $(\mathrm{PC1}=86.5 \%, \mathrm{PC} 2=10.9 \%)$. Figure 7 displayed PCA scores plot of the whole 80 SEM image
TABLE 2: Sensitivity and $1-$ specificity values of classification model using ELM.

\begin{tabular}{lcccc}
\hline Image sample & \multicolumn{2}{c}{ Sensitivity } & \multicolumn{2}{c}{$1-$ specificity } \\
& Calibration & $\begin{array}{c}\text { Cross } \\
\text { validation }\end{array}$ & Calibration & $\begin{array}{c}\text { Cross } \\
\text { validation }\end{array}$ \\
\hline Class 1 & 1.000 & 1.000 & 0 & 0 \\
Class 2 & 0.938 & 0.688 & 0 & 0.016 \\
Class 3 & 0.938 & 0.813 & 0.016 & 0.031 \\
Class 4 & 0.938 & 0.875 & 0.016 & 0.109 \\
Class 5 & 1.000 & 0.938 & 0 & 0 \\
\hline
\end{tabular}

subsamples of the composites in five different aging periods. It is possible to see that image samples of the composites in 20th, 80th, and 100th aging cycles are separated distinctly from each other; although the ones in 40th and 60th aging cycles are a little closer to each other, the observers can still easily identify them by scores on PC2 causing a bit bigger difference between the two neighbor groups.

4.1.2. ELM Classification Results. The main parameters of program implementation of ELM classification were as follows: (1) Application type of training function is set to classification recognition; (2) "train label" takes values from "1" to " 5 " (corresponding to the five different aging periods; namely, train label of 20th aging period of material SEM images is labeled "class1", train label of 40th aging period of material SEM images is labeled "class 2," and so on); (3) type of hidden layer activation function chooses "sig" (Sigmoid function); (4) neurons number of hidden layer was set by cross validation approach after program repetitive running. From repetitive running classification accuracy results of classifiers based on ELM neural network can reach relatively high value when the number of hidden layer neurons approached 17 finally. In order to verify every category classification precision, ROC (Receiver Operating Characteristic) curves of ELM predictive model were used here to evaluate the sensitivity and 1 -specificity values of the predictive model, and the curves of five different categories (corresponding to the five different aging stages) using this model are demonstrated in Figure 8.

Sensitivity and 1 - specificity values of predictive classification model using ELM (with optimal neuron number 17) were reported in Table 2.

4.1.3. SVM Classification Results. The important input parameters of SVM class function were set as follows: (1) "train_label" takes five different values just like the ones in the training function of ELM; (2) $c_{\min }=g_{\min }=-10$, $c_{\max }=g_{\max }=10$; (3) $v$ ( $v$-fold cross validation $)=5$; (4) $c$ step $=g$ step $=0.5$. Optimal parameters of SVM classification model, that is, $c$ (penalty factor) and $g$ (kernel parameter), are determined by cross validation as well. Parameters optimization of this model was realized by using grid search method after running the function SVMcgForClass. According to the found optimal parameters $c$ and $g$, that is, $c=1, g=0.011$, sensitivity and $1-$ specificity 


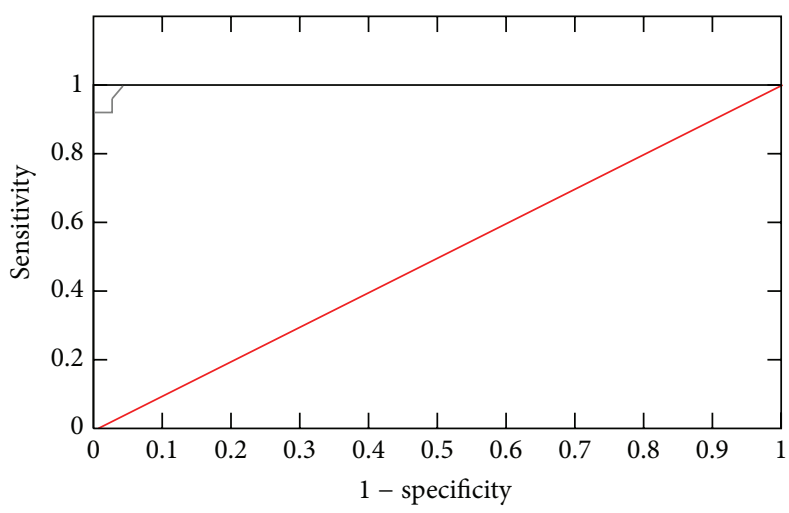

(a) Estimated (black) and cross validation (grey) ROC of class 1

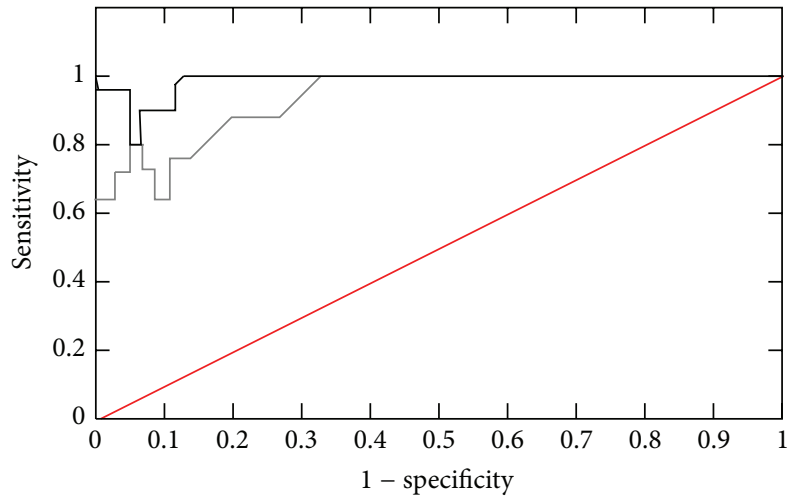

(c) Estimated (black) and cross validation (grey) ROC of class 3

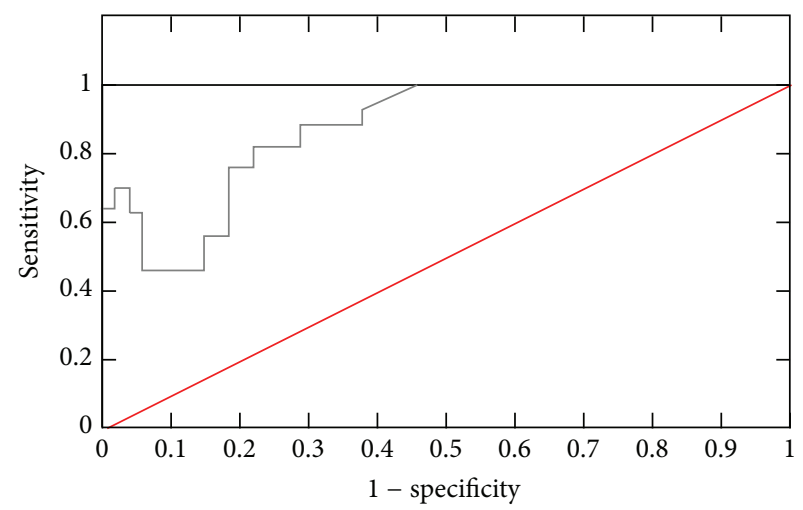

(b) Estimated (black) and cross validation (grey) ROC of class 2

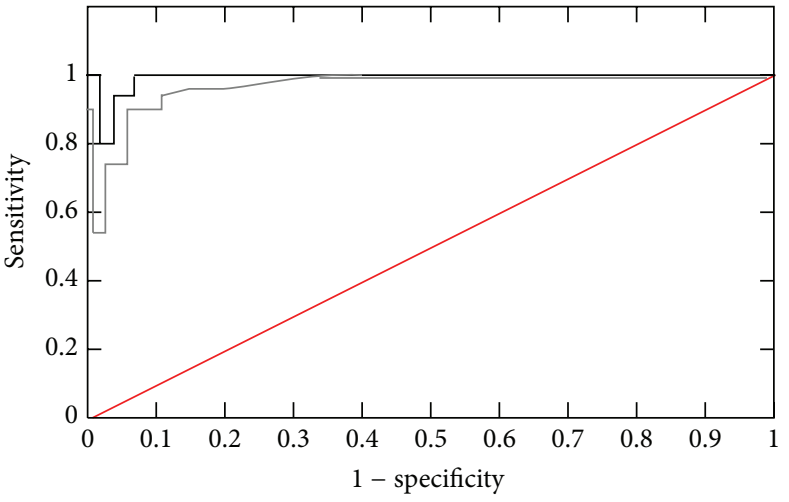

(d) Estimated (black) and cross validation (grey) ROC of class 4

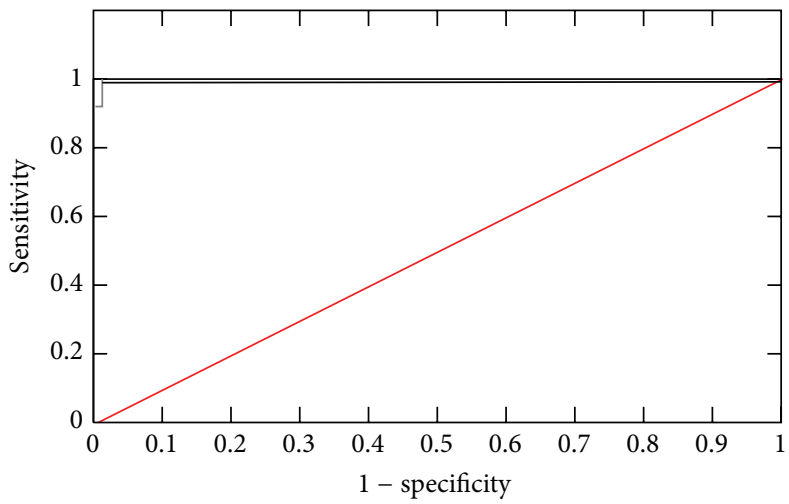

(e) Estimated (black) and cross validation (grey) ROC of class 5

FIGURE 8: ROC curves of ELM classification model of SEM image samples of the composites in five different aging periods.

values ( $c$ changed with fixed constant $g$ ) and their ROC curves of the second predictive model to predict the five different classes are displayed in Figure 9.

Sensitivity and 1 - specificiy values of predictive classification model using SVM (with optimal parameters $c$ and $g$ ) were reported in Table 3.

In order to evaluate and compare the predictive ability and performance of two classification models as a whole, statistics results of overall classification accuracy of model using ELM and SVM are reported in Table 4. Due to the randomness of the weight matrix values, the results of ELM class prediction were a little different every time after program running, so the averaged five preferable running results were taken into account.

4.2. Discussion. From Tables 2 and 3, it is obviously seen that sensitivity values of SVM prediction model were always higher than those of ELM model with respect to the five different classes. For example, sensitivity values of class 2, class 3 , and class 4 using SVM model were $0.813,0.876$, and 1.000 , respectively, while the values of those three classes using ELM model were only $0.688,0.813$, and 0.875 . Meanwhile, 1 -specificity values of the SVM model were commonly lower than that of ELM model; for instance, 1 - specificity values of 


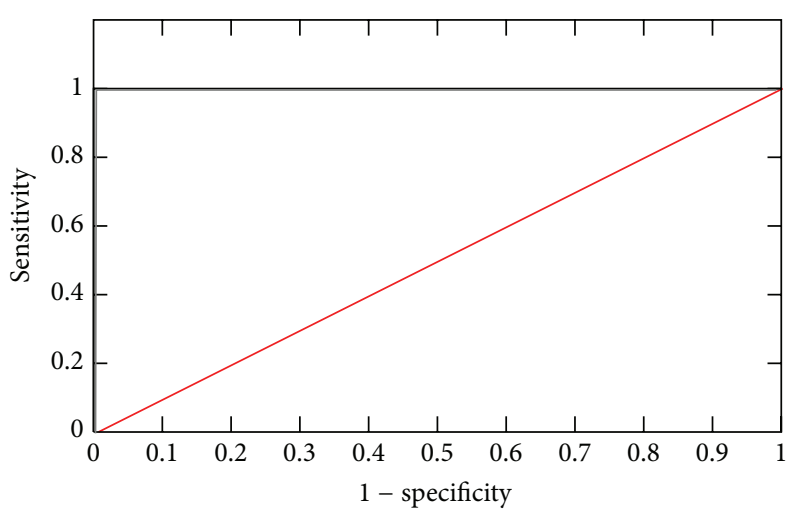

(a) Estimated (black) and cross validation (grey) ROC of class 1

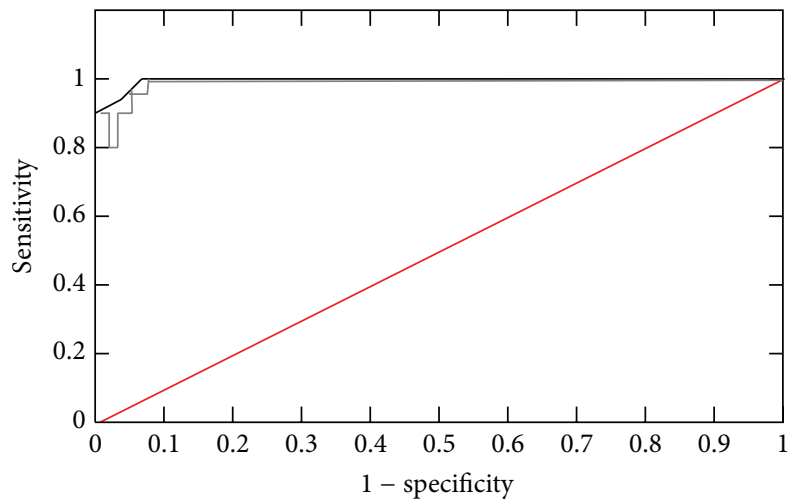

(c) Estimated (black) and cross validation (grey) ROC of class 3

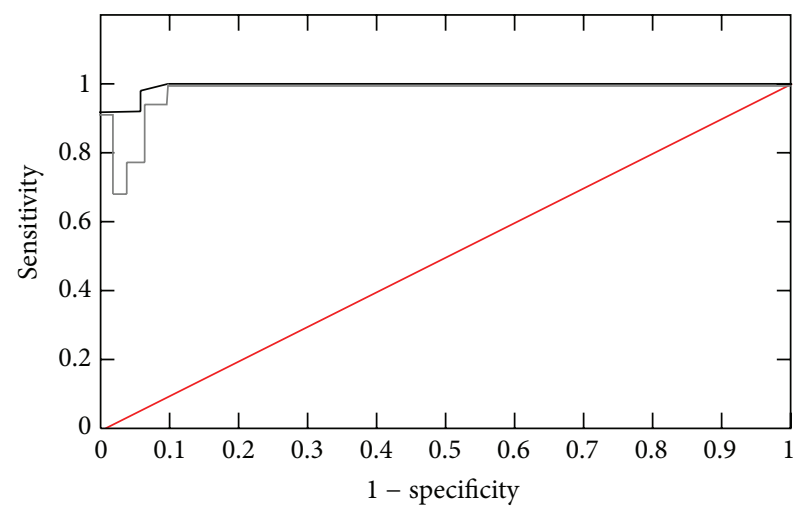

(b) Estimated (black) and cross validation (grey) ROC of class 2

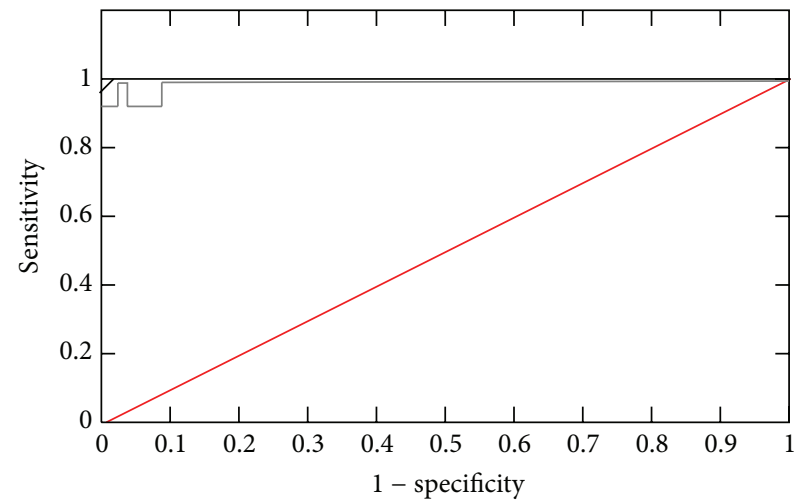

(d) Estimated (black) and cross validation (grey) ROC of class 4

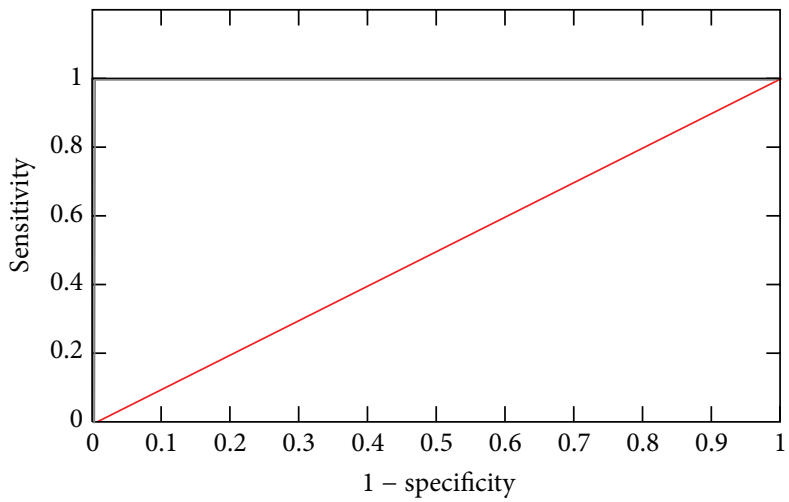

(e) Estimated (black) and cross validation (grey) ROC of class 5

FIGURE 9: ROC curves of SVM classification model of SEM image samples of the composites in five different aging periods.

the three middle classes using SVM model were only 0.016 , 0.047 , and 0.016 , respectively, and nevertheless the values of the same three categories using ELM reached 0.016, 0.031, and 0.109 . It was the true case that predictive classification performance of SVM model is better than that of ELM model for each class to be identified. Among the five different classes especially the middle three classes, that is, class 2, class 3 , and class 4 , it was often the case that, by using SVM model, there is only one adjacent aging period gap between the true categories and estimated ones. For example, the true category of the sample image was "class 2," while predictive category of it was "class 3."
Relating to the overall classification results, reported in Table 4, it can be obtained that averaged classification accuracy of calibration dataset using ELM can be $91.5 \%$, and that of validation dataset was $86.3 \%$. Classification accuracy of the classifier using SVM was relatively higher than that of ELM; its averaged classification accuracy of calibration dataset was $93.8 \%$, and that of validation dataset reached $92.5 \%$.

With regard to the first classifier, from the program running results of the classification using ELM neural network, it can be known that this classifier can achieve acceptable classification accuracy. In ELM neural network classification, 
TABLE 3: Sensitivity and $1-$ specificity values of classification model using SVM.

\begin{tabular}{lcccc}
\hline Image sample & \multicolumn{2}{c}{ Sensitivity } & \multicolumn{2}{c}{$1-$ specificity } \\
& Calibration & $\begin{array}{c}\text { Cross } \\
\text { validation }\end{array}$ & Calibration & $\begin{array}{c}\text { Cross } \\
\text { validation }\end{array}$ \\
\hline Class 1 & 1.000 & 1.000 & 0 & 0 \\
Class 2 & 0.942 & 0.813 & 0 & 0.016 \\
Class 3 & 0.961 & 0.875 & 0 & 0.047 \\
Class 4 & 1.000 & 1.000 & 0 & 0.016 \\
Class 5 & 1.000 & 1.000 & 0 & 0 \\
\hline
\end{tabular}

TABLE 4: Statistics results of overall classification accuracy of model using ELM and SVM.

\begin{tabular}{lcccc}
\hline & \multicolumn{2}{c}{ Calibration dataset } & \multicolumn{2}{c}{ Validation dataset } \\
Classifier type & $\begin{array}{c}\text { Standard } \\
\text { deviation }\end{array}$ & Average & $\begin{array}{c}\text { Standard } \\
\text { deviation }\end{array}$ & Average \\
\hline ELM & $6.3 \%$ & $91.5 \%$ & $7.2 \%$ & $86.3 \%$ \\
SVM & $4.7 \%$ & $93.8 \%$ & $5.6 \%$ & $92.5 \%$ \\
\hline
\end{tabular}

the number of hidden layer neurons of training function is a very important parameter, and with regard to ELM classifier this parameter value mentioned above has a very significant impact. It showed that through training function repeatedly running as the number of the hidden layer neurons was close to 17 relatively high classification accuracy can be achieved. However, continuing to increase the number of the hidden layer neurons, classification prediction accuracy of test set samples would not increase significantly consequently. As for the second classifier, from the program running results of the classifier using SVM, it can be seen that the classifier can obtain higher classification accuracy than the former. In other words, the number of misjudged samples by using SVM model was a little lower than that of ELM. In view of the classification prediction accuracy, accuracy of ELM was slightly lower than that of SVM actually, and for the goal of getting a higher classification accuracy determination of the number of hidden layer neurons using ELM classifier needs to run program repetitively, and it may take a very long time. However, the number of called function and optimal parameters of ELM classifier were much less than that of SVM, so its parameter setting process was relatively simpler and faster.

\section{Conclusions}

In summary, research results in this paper indicate that AMT method can well describe the textural feature of SEM images of microscopic surface topography of the composite. AMT method is capable of expressing discriminating and quantitative characterizations of texture complexity of SEM images. The feasibility and effectiveness of textural feature extraction using AMT method has been proved. According to extracted AMT spectra of SEM images of wheat straw/polypropylene composites in five different aging periods, two classifiers based on ELM and SVM were applied to identify those different aging stages. For the two models SVM model performed better than ELM model in general according to the sensitivity and 1 - specificity values and ROC curves and the overall classification accuracy. Classification approach of SEM images by using AMT spectra and classifier based on this complexity scale data has been verified. It can be expected that growing applications based on AMT method, including research and development of new type composite material, will be increasingly and intensively investigated in more and more aspects.

\section{Conflict of Interests}

The authors declare that there is no conflict of interests regarding the publication of this paper.

\section{Acknowledgments}

This work was supported by the Funded Projects of "Twelfth Five-Year" National Science and Technology Support Plan (Grant no. 2011BAD20B03-02), Basic Scientific Research Special Funds for the Central Universities (no. KYZ200921), and Open Project of Jiangsu Key Laboratory of Large Engineering Equipment Detection and Control (no. JSKLEDC201204). Particularly, sincere and deep thanks are due to Professor K. Kvaal who gave the authors so much guidance and inspiration continually, and it has never been changing that his help finds the authors well and timely always.

\section{References}

[1] R. Selden, B. Nystrom, and R. Langstrom, "UV aging of polypropylene/wood-fiber composites," Polymer Composites, vol. 25 , no. 5, pp. 543-553, 2004.

[2] J. S. Fabiyi, A. G. McDonald, M. P. Wolcott, and P. R. Griffiths, "Wood plastic composites weathering: visual appearance and chemical changes," Polymer Degradation and Stability, vol. 93, no. 8, pp. 1405-1414, 2008.

[3] N. M. Stark and L. M. Matuana, "Characterization of weathered wood-plastic composite surfaces using FTIR spectroscopy, contact angle, and XPS," Polymer Degradation and Stability, vol. 92, no. 10, pp. 1883-1890, 2007.

[4] Y. X. Zhou, F. Pervin, S. Jeelani, and P. K. Mallick, "Improvement in mechanical properties of carbon fabric-epoxy composite using carbon nanofibers," Journal of Materials Processing Technology, vol. 198, no. 1-3, pp. 445-453, 2008.

[5] D. S. Raimundo, P. B. Calíope, D. R. Huanca, and W. J. Salcedo, "Anodic porous alumina structural characteristics study based on SEM image processing and analysis," Microelectronics Journal, vol. 40, no. 4-5, pp. 844-847, 2009.

[6] J. A. Galloway, M. D. Montminy, and C. W. Macosko, "Image analysis for interfacial area and cocontinuity detection in polymer blends," Polymer, vol. 43, no. 17, pp. 4715-4722, 2002.

[7] R. Andrle, "The angle measure technique: a new method for characterizing the complexity of geomorphic lines," Mathematical Geology, vol. 26, no. 1, pp. 83-97, 1994.

[8] J. Huang and K. H. Esbensen, "Applications of angle measure technique (AMT) in image analysis: part I. A new methodology for in situ powder characterization," Chemometrics and Intelligent Laboratory Systems, vol. 54, no. 1, pp. 1-19, 2000. 
[9] U. G. Indahl and T. Næs, "Evaluation of alternative spectral feature extraction methods of textural images for multivariate modeling," Journal of Chemometrics, vol. 12, no. 4, pp. 261-278, 1998.

[10] S. Kucheryavski, "Using hard and soft models for classification of medical images," Chemometrics and Intelligent Laboratory Systems, vol. 88, no. 1, pp. 100-106, 2007.

[11] L. Fongaro and K. Kvaal, "Surface texture characterization of an Italian pasta by means of univariate and multivariate feature extraction from their texture images," Food Research International, vol. 51, no. 2, pp. 693-705, 2013.

[12] R. L. Hou, C. X. He, J. Xie, Y. Min, and D. Chuanchuan, "UV accelerated aging properties of wheat straw/PP wood plastic composite," Acta Materiae Compositae Sinica, vol. 30, no. 5, pp. 86-93, 2013.

[13] S. V. Kucheryavski, K. Kvaal, M. Halstensen et al., "Optimal corrections for digitization and quantification effects in angle measure technique (AMT) texture analysis," Journal of Chemometrics, vol. 22, no. 11-12, pp. 722-737, 2008.

[14] J. Luo, C.-M. Vong, and P.-K. Wong, "Sparse bayesian extreme learning machine for multi-classification," IEEE Transactions on Neural Networks and Learning Systems, vol. 25, no. 4, pp. 836843, 2014.

[15] X. Lu, H. Zou, H. Zhou, and L. Xie, "Robust extreme learning machine with its application to indoor positioning," IEEE Transaction on Cybernetics, 2015.

[16] G.-B. Huang, Q.-Y. Zhu, and C.-K. Siew, "Extreme learning machine: theory and applications," Neurocomputing, vol. 70, no. 1-3, pp. 489-501, 2006.

[17] G. Feng, G.-B. Huang, Q. P. Lin, and R. Gay, "Error minimized extreme learning machine with growth of hidden nodes and incremental learning," IEEE Transactions on Neural Networks, vol. 20, no. 8, pp. 1352-1357, 2009.

[18] V. Cherkassky and Y. Q. Ma, "Practical selection of SVM parameters and noise estimation for SVM regression," Neural Networks, vol. 17, no. 1, pp. 113-126, 2004.

[19] J. Ma, J. Theiler, and S. Perkins, "Accurate on-line support vector regression," Neural Computation, vol. 15, no. 11, pp. 2683-2703, 2003. 

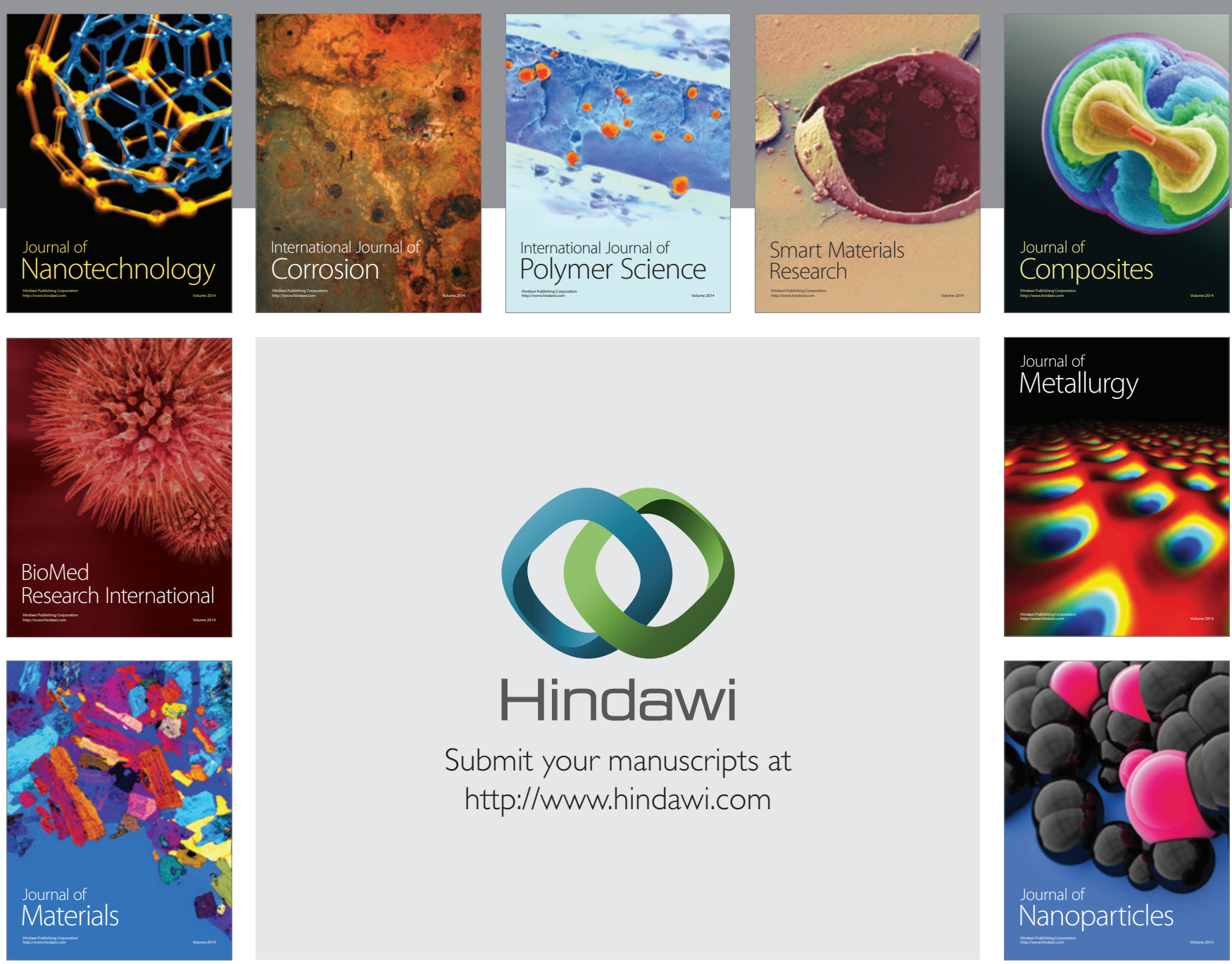

Submit your manuscripts at http://www.hindawi.com
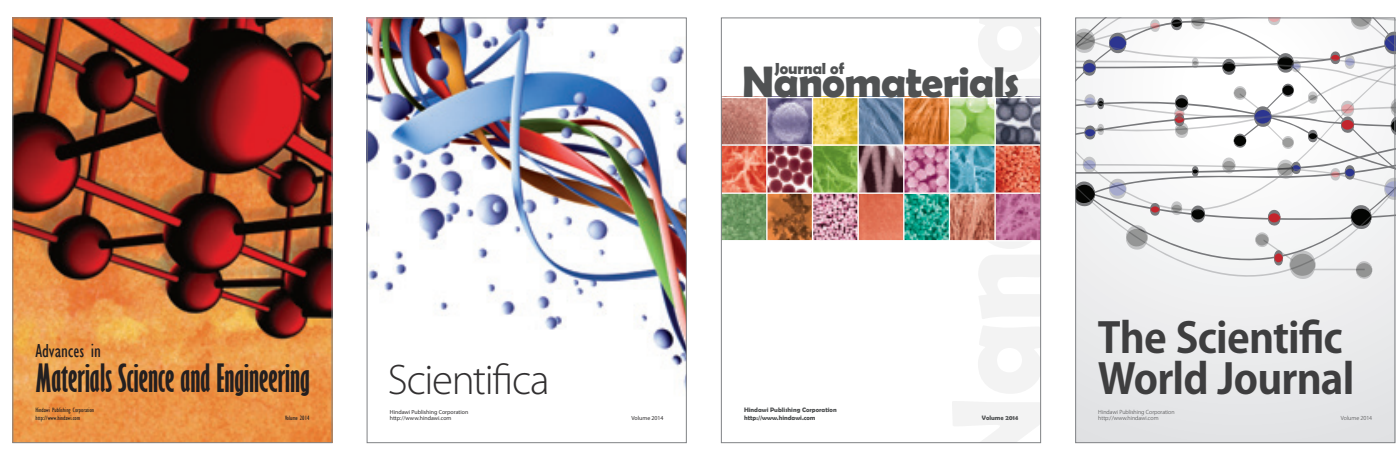

\section{The Scientific World Journal}
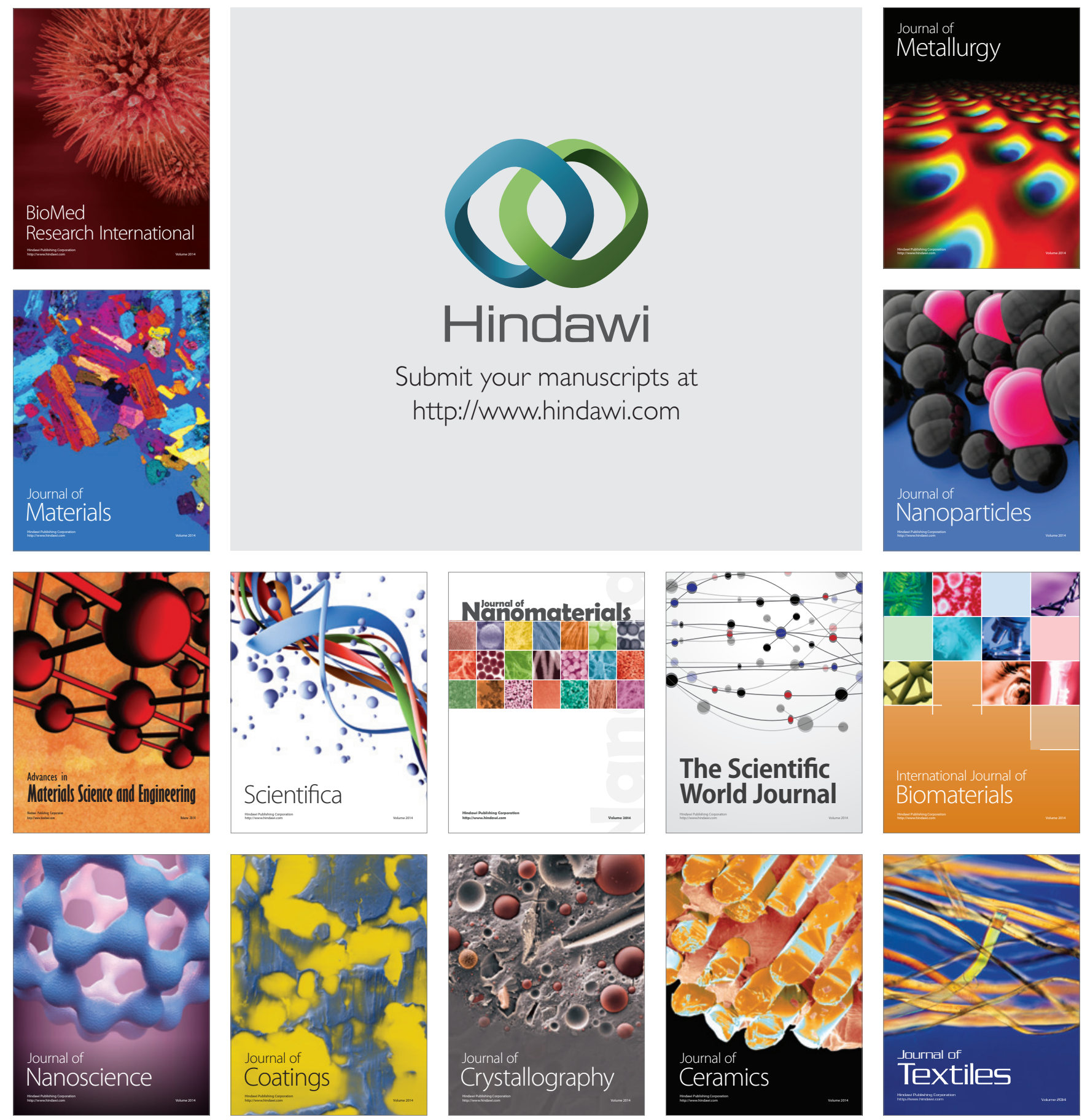